\title{
The Bai Language: A Musical Language with Typological Ablative Cases
}

\author{
Suqin Li ${ }^{1,2}$ \\ ${ }^{1}$ School of Foreign Languages of Southwest University, Chongqing, China \\ ${ }^{2}$ School of Foreign Languages of Dali University, Dali, China \\ Email: li_suchin666@163.com
}

Received 3 December 2015; accepted 26 December 2015; published 29 December 2015

Copyright (C) 2015 by author and Scientific Research Publishing Inc.

This work is licensed under the Creative Commons Attribution International License (CC BY). http://creativecommons.org/licenses/by/4.0/

c) (i) Open Access

\begin{abstract}
The Bai language (shortened as Bai henceforth) is of Sino-Tibetan language family, used in southwest China. Typological ablative cases are found in Bai that the word-building in some dialects follows a few archaic analytical rules, including the formation of its pronoun system, its antonymic verbs and adverbs, and the formation of its tetra-syllabic phrases, etc. The most particular feature is that the morphological change in the antonymic verbs realizes the grammatical function. The archaic formations may be remnants of Yi-Burman proto language, which needs historical studies in terms of language evolution. However, the pivotal motivation, we believe, is the need to be a musical language in satisfying the various needs to transmit the ethnic culture orally, and this need is the critical stimulus to make it keep the inflective changes.
\end{abstract}

\section{Keywords}

Bai, Musical Language, Typological Ablative Case, Oral Transmission, Grammatical Function

\section{Introduction}

Morphology is one of the critical sub-branches in the classic typology studies. From the angle of morphology, languages are classified as analytic, isolated, agglutinant, and polysynthetic according to their morphological features [1]. The distinctions among the types are a matter of probability. Therefore, we are not surprised to see some morphological changes functioning grammatically in the Bai language (Bai in short hereafter), an isolated language used in southwest China. Bai is spoken by ethnic people with a population of around two million, who live in the areas around the Erhai Lake in Dali prefecture of Yunnan province, with a few sparsely living in other places such as Guizhou and Hunan province. Bai has seven to nine tones, and has a few sound and tone variations among the three subdivisions of dialects. These make the language a musical one. People amuse the way 
Eryuan people do self-introduction, as they would say wo shi er yuan ni "I am from Eryuan" in a tune like singing the notes "duo suo duo suo mi, 15153 ".

In our investigation into the systemic functional grammar profile, we encounter three interesting ablative cases in the word-building. We assume that the music-like feature of the language has evolved as being motivated partly by the external needs, and becomes possible in the internal features of the mono-syllable language per se. We guess that some of the features are typologically universal in Tibeto-Burman language family [2]; some might be the result of geographical diffusion. And some might be remnants of a proto language. The primary motivation of the musical features, we believe, is to meet the need of oral transmission of its wisdom, skills, techniques, rituals, literature works, labor-sharing and organization, and serve other aspects of the community life.

Bai is divided into three regional dialects, namely, the northern dialect, the middle dialect and the southern dialect. The middle and southern dialects are intelligible to the speakers in both regions, but have trouble with the northern dialect speakers. The study is based on data collected in Niujie, a dialect spoken in the middle subdivision of the three major dialects of Bai. Niujie Bai is found to be rich with many other archaic grammatical features, and more discussion will be published in other places.

\section{Inflective Morphological Changes}

The inflective word-formation is found in the pronoun system, the verbal system and the four-syllabic formations.

\subsection{The System of Pronouns}

In Table 1, the mark “ " represents one copy of the word in the upper case above it in the table. Now we find out the regular changes corresponding between Row 2 Nominative and Row 3 Accusative in the singular pronouns. The vowel $o^{31}$ in the three singular pronouns $\eta o^{31}, n o^{31}, \mathrm{mo}^{31}$ changes to $\mathrm{u}^{55}$ with the tone changing from mid-falling 31 to high-level 55, $\eta i^{55}$ remains regular change by adding one suffix $-\eta v^{31}$, like in other persons. This suffix $-\eta v^{31}$ is a bound morpheme, which cannot stand alone to be one word. It sequences a personal pronoun to make it accusative.

\subsection{The System of Antonym in Verbs}

As one of the salient features of polarity in systemic functional typology, the expression of "negative" polarity may be a negative verbal affix [3]. The particular way of the negation in Bai is in its inflective changes in morphemes in a few verbs, adverbs, and adjectives, resulting in pairs of antonyms. The specific changes may occur to the vowels and form such an insertion of mediate vowels like $u$ and $i$, or a change of the vowel from $u$ to $o$, or changes occurring to the consonants like from $\eta i^{44}$ to $\mathrm{mio}^{44}$, from $\mathrm{tu}^{44}$ to $t s h w^{55}$ with tone rising shift from the mid-level tense 44 to the high level lax 55 .

A few verbs have inflective changes to get their antonyms and therefore the negation is actualized with the negation on the verb. See the following examples and observe the words in bold.

1) A: $n o^{31} \boldsymbol{s e}^{33} \mathrm{mo}^{31} l \varepsilon^{35}$ ? "Do you know him?"

B: $m u^{55} n \supset^{33}\left(\eta o^{31}\right) \boldsymbol{a}^{35}$ sue $\boldsymbol{e}^{33}$. "Him (I) don't know."

him (I) NEG NEG know

This example contains a pair of verbs $s e^{33}$ "know" and $s u e^{33}$ "not know". But the following pair of antonyms may arouse confusion in terms of its function.

Table 1. Nominative and accusative pronouns.

\begin{tabular}{|c|c|c|c|c|c|c|c|c|}
\hline Person/Number & 1/Sgl & 1/Plr/Exc & 1/ Plr/Inc & 2/Sgl/fm & 2/Sgl/pol & 2/Plr & 3/Sgl & 3/Plr \\
\hline Nominative & $y o^{31}$ & $y a^{55}$ & $\eta \operatorname{lia}^{55}$ & $n o^{31}$ & $\eta i^{55}$ & $n a^{55}$ & $m o^{31}$ & $m a^{55}$ \\
\hline Accusative & $\eta u^{55} \eta v^{31}$ & $\sim \eta v^{31}$ & $\sim \eta v^{31}$ & $\eta u^{55} \eta v^{31}$ & $\sim \eta v^{31}$ & $\sim \eta v^{31}$ & $m u^{55} \eta v^{31}$ & $\sim \eta v^{31}$ \\
\hline
\end{tabular}


2) A: $n a^{55} \eta i^{33} \eta o^{31} t a^{33} n a^{55} \mathrm{~S}^{31} \eta a^{21} l \varepsilon^{55}$ ? "Do you want me to go with you?"

you want me with you go MOD ADJ

B: $t s i^{31} t_{s o}{ }^{4_{2}} . \quad y a^{55} \boldsymbol{a}^{35} \mathbf{m i o}^{33}$. "No, we don't want to."

No need we NEG NEG want

$\eta \mathrm{io}^{33}$ and $\mathrm{mio}^{33}$ are used as a pair of antonyms in this context. Other pairs of antonymic verbs are $t \tilde{a}^{42}$ "be able

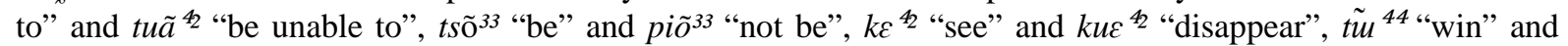
tshíu "lose" (esp. in gambling) etc. Many other pairs of such verbs are spoken by old people but unknown to younger generations by and by. These pairs of verbs are used more often in Niujie than in Jianchuan, less heard of in southern dialect around the Erhai Lake [4].

\subsection{Antonyms of Verbal Adverbs}

Antonyms of verbal adverbs are limited in number but used in high frequency.

3) A: $k^{55} \tilde{j l}^{33} \mathrm{no}^{31} \mathrm{ga}^{21} \mathbf{s i}^{55} \mathrm{tso}^{42} \mathrm{mo}^{44}$ ? "Will you go or not today?"

Today you go will or not?

B: $\eta o^{31} \quad y a^{21} \mathrm{mo}^{3}{ }^{1} \mathbf{s u}^{55}$. "I don't want to go."

I go NEG NEG will / I not go will

In this pair of adverbs $s i^{55}$ and $s u^{55}$, the vowel $i$ changes to $u$. In other pairs of antonyms, vowel insertion happens, or consonant being aspirated.

4) A: $a^{31} t^{55} \mathrm{mo}^{33}, s^{33} f y^{31} \quad \quad \lim ^{31} t^{44} \quad j i u^{33} \mathrm{tsi}^{31} \mathrm{xu}^{33}$ ? “Aunt, is this mushroom safe to eat?” aunt, mushroom this MEAS eat

B: $j i u^{33} \mathrm{xo}^{33 !} \quad m o^{31} \quad t o^{31} \eta i^{21} k^{55}$. "You can’t eat it! It poisons people." eat can't it poison person

$\mathrm{ta}^{55} \mathrm{mo}^{33}$ originally is a specified address for one's uncle's wife, but it can be enlarged to address any woman at one's mother's age as $a^{31} \mathrm{ta}^{55} \mathrm{mo}^{33}$. This practice is used in Korean too [5].

\subsection{The Inflective Change in the Tetra-Syllabic Formations}

Duplication is one way of word-formation as the following two examples.

5) $l o^{21}$ "tiger" $+p a^{42}$ "leopard" $=l o^{21} l o^{21} p a^{42} p^{42} \quad$ "fierce wild animals" [6] $m i^{33}$ "miss" + $k a^{31}$ "plan" $=m i^{33} \mathrm{mi}^{33} \mathrm{ka}^{31} \mathrm{ka^{31 }}$ "think carefully and plan again and again"

And tetra-syllabic is formed via partial duplication. E.g.:

6) $k u^{44} \eta i^{21} s e^{3 l} \eta i^{21} \quad$ "all people, the old or the young" old person small person

7) jo ${ }^{31} l^{31} \mathrm{pi}^{44}{ }^{4} \tilde{u}^{44}$ "shaking and shivering" shake shiver ( $l o^{31}$ and $l i \tilde{u}^{44}$ are inserted syllables with no meaning)

Tetra-syllabic is found to be a common word formation in Sino-Tibetan languages [7]. According to the prolific documents of minority languages spoken in northwestern areas of Yunnan Province, Bai, Naxi, Dulong, Pumi, Nusu and Yi languages, are found to have the tetra-syllabic formations in large amount. Used in daily talks, folklore telling, and folk songs, these formations are vivid and brief. And users tend to be those elder people who have not received Chinese education, who can speak their mother language every day, or those native speakers who live in the rural or mountainous villages.

\subsubsection{Zero Inflective Change}

In Bai, there are Measures which are converted from the nouns, which we term as zero inflective change.

8) $j i m^{44}\left(a^{31}\right) \quad j i u^{44}$ one village (village) (one) (village MEA)

$\operatorname{ta}^{4_{2}}\left(a^{31}\right) \mathrm{ta}^{4_{2}}$ "a load of (goods or crops)" (load) (one) (load MEA)

$k v^{55}\left(a^{31}\right) \quad k y^{55}$ "one river" $\eta i^{21} k \varepsilon^{55}\left(a^{31}\right) \quad \eta i^{21} \quad$ "one person”

(person) one (person MEA)

$t^{21} \quad\left(a^{31}\right) \quad t a^{21}$ "one patch of farming land"

(patch) (one) (patch MEA)

$k o^{31}\left(a^{31}\right) k o^{31}$ "one lake" 


$$
\text { (river) (one) (river MEA) ～(lake) (one) (lake MEA) }
$$

If we need enunciate it as "one" of something, we insert the numeral $a^{31}$. Otherwise, the structure of " $\mathrm{N}+\mathrm{N}$ " is understood as one measure of $\mathrm{N}$. "Two rivers" is $k v^{55} \mathrm{ko}^{33} k v^{55}$ (river two river) "two rivers". In the construction, the first noun is a countable noun, and the duplicate of this noun becomes its measure to modify the first noun. Such "Noun + (Numeral) + Measure (being copy of the Noun)" structure is similar to that in Burmese nominal phrases with measures. We cite examples by Wang Danian (Wang, 2002) [8].

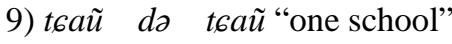
(school) one (school MEA)

jwa do jwa "one village” (village) one (village MEA )

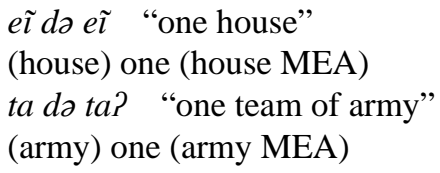

$\mathrm{N}_{1}$ and $\mathrm{N}_{2}$ are the same phrase with the same tone, but in both languages, $\mathrm{N}_{2}$ becomes a measure. These changes can be regarded as "zero inflective change" since they realize their function merely by the collocation sequencing.

\subsubsection{Superasegmental Changes}

We have illustrated two kinds of inflective changes. The first one is realized with the replacement of vowels, and the second one is "zero inflective change", realized merely by the sequencing of the copy of the noun to make it a measure specifically for this noun. The third kind of inflective change occurs to the tones as the examples below, and we will name it "superasegmental inflective changes". This inflective change occurs to a few nouns to convert them to verbs, or, maybe in the opposite direction, from verbs to nouns.

10) $t a^{55} a^{31} \quad t^{42}$

or in short $\quad \mathrm{ta}^{55} \mathrm{ta}^{42} \quad$ “carry a load/duty on one's shoulder”

$\mathrm{V}$ one load

11) $v y^{33} a^{31} \quad v y^{33}$ or in short $v y^{33} v y^{33}$ "carry a load on one’s back"

$\mathrm{V}$ one load

12) $j^{4_{2}} \quad j i^{55} \quad$ "wear clothes (upper dress)"

$\mathrm{V}$ coat/blouse

In these examples, the tone change leads to the conversion of the part of speech, i.e. the morphological changes alter the function of the first phrases in these pairs of phrases.

Similar features can be found in other Tibeto-Burman languages, take Lahu language for example. This rule seems a reasonable explanation for the motivation of Tibeto-Burman languages, which are mostly monosyllabic and ending with vowels, and incidentally, have large amount of four-syllabic languages [2]. Lahu language has harmonic sound system, manifesting similar inflective morphological change rules just like that occurring to the Bai language. Therefore researchers like Liu Jingrong [9] holds that analytic and monosyllabic languages tend to generate four-syllabic words. To this idea, Bai stands true. Most of the languages in Tibeto-Burman family are generally mono-syllabic and are tone languages.

Morphological changes occur to Bai to realize grammatical functions. We find interesting parallel situations in Burmese [8]. Burmese is also classified an isolated language, in which morphological changes are rare, with a few ablative cases, in which, morphological changes function grammatically via inflective changes. In Burmese, tone changes, stress changes and vowel or consonant replacement may function to meet grammatical requirements. To illustrate this, we cite the following Burmese examples given by Wang Danian and give it our explanation according to the citations.

To convert a verb to a noun, the voiceless consonants are changed to be voiced, and the stressed vowel [a] turn to be unstressed [ə]. e.g.:

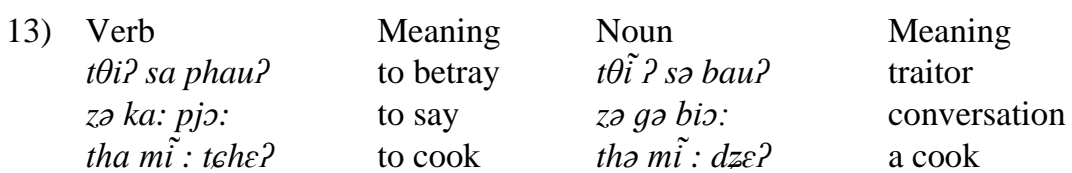

To make possessive relation, Burmese reduces the particle $i$, and adds the location of the tone on the particle $i$ to the pronoun itself. e.g.: 
14) t6ว nо $i^{\prime} \quad \rightarrow$ ni my Poss Mark brother $t \theta u \quad i^{\prime} \quad \tilde{i}: d z i \longrightarrow$ his Poss Mark clothes

t6ə nə'
my Poss Mark brother
$t \theta u^{\prime} \quad \tilde{i}: d z i$
his Poss Mark clothes

Comparing these cases in Bai and Burmese, we tend to believe the reflective changes in Bai are probably remnants of the archaic language forms.

\section{Motivations of the Inflective Changes}

\subsection{To Meet the Need of the Farming Working Style}

The lack of a writing system for the mass media use is a lamentable check to the diffusion of knowledge for a community. The only reliable channel is oral recitation and passing down to the younger generations. This primary demand sets a precondition - the words and phrases in Bai must be harmonious with the melody, tonerich and easy for a better and quicker memorizing of those orally transferred techniques and skills.

The musical feature of the language makes it easy to organize the population. Farming, fishing and craftsmaking, esp. silver ornaments and wood carving, are the major ways by which Bai people make a living. It is a regular practice to share labor when in sowing and harvesting seasons to rush for the timing working. The share-labor system exists till today in farming and other household work, for instance, sharing labor in building up a mud wall, cooking together in treating guests for weddings or funerals or other significant household events. Language is used here as the instrument of organizing and entertaining the people at work.

\subsection{To cater to the Needs of Musical Expressions of Emotions and Feelings}

Meanwhile, the lack of a writing system stimulates the creation of a huge amount of oral literature works, including art works in the singing forms of Dabenqu (Bigbook Tunes, a narrative story told by singing), Duige (Songs Replying, collective improvisation in asking and answering in singing between men and women on festival occasions, or between young people at courting places) and Tasangge (Song of Stepping on the Funeral, lamenting the dead by singing while stepping around the coffin) [10], in conveying the wisdom, the humor and ideology of the Bai nationality. The inflective changes help make the lexemes more musically harmonious. The musical words and phrases enable the oral cultural works to be refined, stored, memorized, and spread.

In the life of many minority nationalities, music is indispensable on many occasions. For instance, on ceremonial occasions, and other rituals, singing is a necessary practice. In the funeral of the Bai nationality, the family members of the dead must practice Ta-sang-ge [10], meaning stepping around the coffin and singing to the dead. In this singing, family member recall his lifetime and express their sad regret of losing him, then pray for him, wish him rest in peace. The man in charge of the ceremony is chosen and respected by the villagers, so he is supposed to judge the children of the dead in his singing words, which are usually improvised at the spot. If they treated their parent bad before his passing away, the man may criticize the children on this occasion, when most of the villagers and relatives collect in the yard to help shou-ye "guard the night" for the dead. On the day when the coffin is sent to bury, the team of men carry the coffin and followed by women family members, who usually haul or cry aloud with words to say their last words to the dead as Bai people believe the newly passed away can hear people in the first seven days, at particular time points, for instance, the time when the cock crows first time in the morning, around $5 \mathrm{am}$. They would wail with lamenting words to the dead while worshipping him with burning incense and setting the table of food, like providing three meals when their parents are alive.

Other forms of musical expression are used in courting and dating among Bai people. For instance, the famous festive event Shi-bao-shan Dui-ge (Stone Jewelry Mountain Singing Festival) in Jian-chuan County and Rao-shan-lin (Circling around the forest, a festival nicknamed as the Bai people's "lover's day" as Bai people can sing love songs to each other on this day) in the Southern dialect area are good time for one to enjoy the rich metaphors and vivid language of Bai. The young man is judged to be good enough to marry if he is eloquent at the wording and quick to respond with wit and sincerity. Bai people believe that a singer cannot lie in his singing words. The moment when he is singing, it is the good time to check whether he is trustworthy, reliable enough to be a good husband. This event makes the thing of dating full of wisdom and, inevitably, full of the fierce verbal competition when more than one young man loves the same girl. The singing is usually accompa- 
nied by a musical instrument named Sanxian, at regular beats and to certain tunes. This requires words and sentences should be melodic and even to the tune. There is no writing system for villagers to use to compose the words in advance. And the singing needs response at the spot. The trying of one's verbal ability is stressful and the demand for the smoothness and forcefulness of his words is apparent. Objects, things in the nature around him could be good material for the singer to use in the poetic and metaphoric wording.

Therefore, we claim that the inflective changes occurring to Bai language is a consequence of the musical language to meet the needs to be prosodic and musical. Music becomes more moving with the words it accompanies and words become more expressive under the help of music. The form of language is closely related with music [11].

\subsection{Result of Social Communication}

In most of the ethnic societies, where no common written words are in everyday life use, the social members engaged in social activities such as labor-sharing need effective oral language to organize the shared-working. Sharing labor remains a usual practice in farming and producing handcraft products in Bai area till today. In such tradition, language can serve the social function at three layers.

Verbal activities are applied to assign work and communicate information, such as handing down the skills and techniques of farming or craftwork making. Music, or verbal activities accompanied by musical instruments such as "Sanxian (a three-stringed guitar)" can help increase the unity and give incentive to people at hard labor.

The communication is also carried between the human and the gods. In reciting the scriptures, the old women would sing the words to a certain tune. The man in charge of the rituals usually is good at singing because he needs conduct his ceremony procedures while singing to explain the instructions to the mass. The language used to talk with the gods need brief wording and pleasant phonemes to hear. The sparse prosodic words are full of the philosophy and ideology thinking and values toward the world. Tunes and ballads make a major portion in the verbal communication of Bai people, esp. when they are talking with the gods, the nature, their forefathers, and their beloved ones in Duige.

The last kind of musical verbal activity we want to introduce is Dabenqu, the ballad and folklores remembered, retold and passed from mouth to mouth. These Qu, or tunes, are oral literature works, on a wide range of topics, including a woman's language missing for her husband who has gone far away to earn money; or women's sad story of being maltreated by their mother-in-law, or women's sad story of having a gambler husband, or stories teaching people goodness from the evil. Singing with strong emotion, the singer of the stories moves her sentimental listeners to cry. Thirty years ago, the singing and appreciating of Dabenqu together was a social gathering in rural life, when television sets and smart phones have not yet come into villagers' lives to keep them at home and kill their spare time alone.

\subsection{Geographical Diffusion}

In Tibeto-Burman languages, such inflective changes are common. Take the languages spoken in the northwestern Yunnan as example, the universal typological feature is that languages all have tetra-syllabic formation [2], and the pronoun system of them are generally formed via inflective changes. As to verbs, they share similar feature again in that some of the verbs have antonymic counterparts. After a quick check on the fifty prolific introductions to the minority languages in China, we are impressed that more than ten languages of the Tibeto-Burman family are reported to have tetra-syllabic formation. What seemingly coincident is that these languages are spoken by ethnic people who live in northwestern part of Yunnan province. The first explanation is typological universal as it is known these languages are of the same family-Sino-Tibetan family. Besides, we believe in geographical diffusion. Pumi, Naxi, Lisu, Bai, Yi and Tibetan speakers reside over the mountains and in the valleys along the Nujiang Canyon area, Lantsan River area and Hengduan Ranges. Similar geographical surroundings may be part of the causes of the language similarities.

\subsection{Internal Motivations of Inflective Changes}

Finally, the phonetic systems of the tone language makes all the external needs possible. The pivotal internal motivation lies in the fact that Bai is a Tone language, with seven to nine tones in the three subdivisions of dialects. 
The production of musicality via four-syllabic word formation in Bai language is a spontaneous result due to that a majority of Bai lexemes are monosyllabic, and unavoidably words end with vowels, with 7 to 9 tone in the tone system. This condition is surely easy to generate four-syllabic words. The phonetic systems provide a rich soil for the generation of musical sense.

\section{Conclusion}

The language of one nationality construes the experiential into words and sounds. It can go further to establish the real world [12]. The typological universal feature of having the tetra-syllabic formation among the minority languages northwestern Yunnan province testifies to the relation among language, social communication and cognition. There is a relation of co-existing and co-enhancing. To be specific, languages work to facilitate human communication and cognition. On the other hand, social communication and the habit of human cognition enhance the evolvement of languages. The functions of social communication and cognizing the world determine the features of a language. And a minority language needs evolve to be the most musical and suitable for its preservation, its spreading in the duration of its strategic competition with the force of dominant languages. The inflective changes mentioned in this paper illustrate how the morphological changes help realize the grammatical functions in Bai. This is a consequence of the oral language's natural choice in its long-term evolution.

\section{Acknowledgements}

Ministry of Education of the People’s Republic of China, Youth research project (13YJC740046).

\section{References}

[1] Humboldt, W.V. (2004) Uber Die Verschiedenheit Des Menschlichen Sprachbaues und ihren einfluss auf Die Geistige Entwicklung des Menschengeschlechts (Lun Renlei Yuyan Jiegou de Chayi Jiqi dui Renlei Jingshen de Fazhan de Yingxiang) (X. P. Yao, Trans.). The Commercial Press, Beijing.

[2] Li, S.Q. (2014) Investigation of the Types of the Tetra-Syllabic Words of Tibeto-Burman Languages in Northwest Yunnan. Journal of Dali University, No. 9, 51-54.

[3] Cafferal, A., Martin, J.R. and Matthiessen, C.M.I.M. (2004) Language Typology: A Functional Perspective. John Benjamins, Amsterdam/Philadelphia. http://dx.doi.org/10.1075/cilt.253

[4] Wáng, F. (2006) On the Negative Words and Negation Expression in the Bai Language. Journal of Dali University, No. 7, 28-33.

[5] Zhao, J. (2002) The Linguistic Traits of Korean and the Culture of Honorification. In: Ding, W.Y., Ed., Dongfang Yuyan Wenzi Yu Wenhua (Languages, Words and Cultures of the East), Peking University Press, Beijing, 359-373.

[6] Xu, L. and Zhao, Y.S. (1984) Baiyu Jianzhi (A Profile of the Bai Language). Minzu Press, Beijing.

[7] Dai, Q.X. and Sun, Y. (2005) The Mechanism of the Jingbo Tetra-Syllabic Formations and Their Typological Features (Jingboyu Siyin’Geci Chansheng de Jizhi Jiqi Leixingxue Tezheng). Studies of The Chinese Language, No. 5, 432-440.

[8] Wang, D.N. (2002) Burmese Language, words and Burmese Culture. In: Ding, W.Y., Ed., Dongfang Yuyan Wenzi Yu Wenhua (Languages and Cultures of the East), Peking University Press, Beijing, 195-238.

[9] Liu, J.R. and Zhang, Q. (2010) The Semantic Features of the Tetrasyllabic Phrases in the Lahu Language. Minority Languages of China, No. 3, 34-42.

[10] Zhao, M. (2008) An Analysis of “Tasangge”-The Custom of Bai People’s Funeral Elegy. Journal of the Central Univeisity for Nationalities (Philosophy and Social Sciences Edition), No. 6, 131-134.

[11] Kivy, P. (2007) Music, Language and Cognition. Oxford University Press, Oxford/New York.

[12] Halliday, M.A.K. and Matthiessen, C.M.I.M. (1999) Construing Experience through Meaning. Continuum, London and New York. 